\title{
Simulation-Based Design of Production Networks for Manufacturing of Personalised Products
}

\author{
Dimitris Mourtzis ${ }^{*}$, Michalis Doukas, and Foivos Psarommatis \\ University of Patras, Lab for Manufacturing Systems and Automation (LMS), Patras, Greece \\ \{mourtzis, mdoukas, psarof\} @lms.mech.upatras.gr
}

\begin{abstract}
This paper presents a method for the design of manufacturing networks focused on the production of personalised goods. The method, which is implemented to a software tool, comprises of a mechanism for the generation and evaluation of manufacturing network alternative configurations. An exhaustive search and an intelligent search algorithm are used, for the identification of efficient configurations. Multiple conflicting user-defined criteria are used in the evaluation, including cost, time, $\mathrm{CO}_{2}$ emissions, energy consumption and quality. Discrete Event Simulation models of manufacturing networks are simulated for the calculation of performance indicators of flexibility, throughput and work-in-process, and are used for assessing the performance of centralised and decentralised networks. The results obtained through the exhaustive and intelligent search methods are compared. The applicability of the method is tested on a real-life industrial pilot case utilising data from an automotive manufacturer.
\end{abstract}

Keywords: Simulation, Planning, Decentralisation, Personalisation.

\section{Introduction and State of the Art}

The market globalisation trend causes the decentralisation and internationalisation of supply chains and of manufacturing activities [1]. Increased outsourcing is realised by Original Equipment Manufacturers (OEMs) leading to the formation of strong cooperation bonds with their suppliers that have to be coordinated and aligned towards achieving common goals. Innovative production concepts replaced traditional network structures [2]. Moreover, customers demanded personalised products, available at low prices and high quality, at the right time [3]. The demand volatility calls for systems that efficiently deal with uncertainty in inventory planning [4]. Also, globalisation increased $\mathrm{CO}_{2}$ emissions primarily due to production electricity generation methods used [5].

The need for designing and planning efficient manufacturing networks in today's landscape is evident [6]. Current approaches tackle the manufacturing network configuration problem using mathematical programming, bound computation, heuristics, meta-heuristics and sensitivity/stability analysis [7]. Computer simulation,

\footnotetext{
* Coressponding author.

C. Emmanouilidis, M. Taisch, D. Kiritsis (Eds.): APMS 2012, Part I, IFIP AICT 397, pp. 301-309, 2013.

(C) IFIP International Federation for Information Processing 2013
} 
nevertheless, has been indispensable for evaluating what-if scenarios for the design and planning problem of manufacturing networks. Discrete Event Simulation (DES) is a necessary tool in order to assess performance indices of dynamic system behaviour [8][9]. Many approaches have been proposed for dynamic manufacturing network management using simulation techniques. A continuous modelling approach for supply chain simulation was applied in the automotive industry and depicted that initial inventory levels and demand fluctuation can create delivery shortages and increased lead times [10]. A DES approach that included a decision-making mechanism was presented in [11]. However, it did not indicate the best configurations of the supply chain but was utilised for identifying potential solutions. A simulation approach that uses meta-heuristics was tested on a newspaper production and distribution problem. The applicability of the method however, was not validated through a real manufacturing problem [12]. The proposed approach in this paper includes a macroscopic investigation of the performance of manufacturing network measured in terms of flexibility, annual throughout and Work-in-Process (WIP) for the identification of efficient configurations for the manufacturing and transportation of personalised products. Concluding, the proven NP-hard problem of identifying efficient manufacturing network configurations [13] requires intelligent methods in combination with simulation [14].

The contribution of the suggested approach can be found in the following. The decision-making mechanism is tightly integrated with the simulation engine because the best alternative schemes that derive from the first comprise the input to the latter. Moreover, the Intelligent Search Algorithm (ISA) that uses three adjustable control parameters is presented and compared to an exhaustive method. ISA is an artificial intelligent search method that is utilised for identifying high quality solutions in a timely manner. ISA can be valuable in cases when exhaustive search is non-feasible due to the required computational effort introduced by the magnitude of the search space [7][15]. Additionally, centralised and decentralised manufacturing networks are compared regarding their performance under highly personalised product demand. Finally, the method is tested on a real-life industrial case utilising data from an automotive manufacturer.

\section{Manufacturing Network and Product Modelling}

The manufacturing network models under investigation consist of traditional centralised manufacturing network (CMN) structures, where assembly tasks can only be performed by the OEM at specific plants and decentralised manufacturing networks (DMN), where, a set of suppliers and dealers (partners) can perform personalisation tasks (e.g. application of the wrap cast carbon) (Fig. 1) [15].

The personalised product under investigation is a hood and a door of a commercial car. The personalisation options are the addition of a custom sticker, a personalised image and a cast carbon wrap. Fig. 2 contains the Bill of Materials (BoM), the Bill of Processes $(\mathrm{BoP})$ and the different Levels of Personalisation (LoP) used in the experiments. 


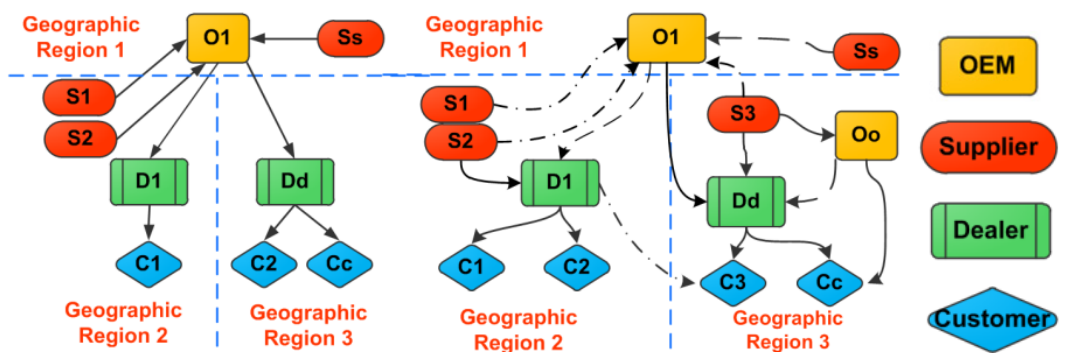

Fig. 1. Models of centralised and decentralised manufacturing networks [15]

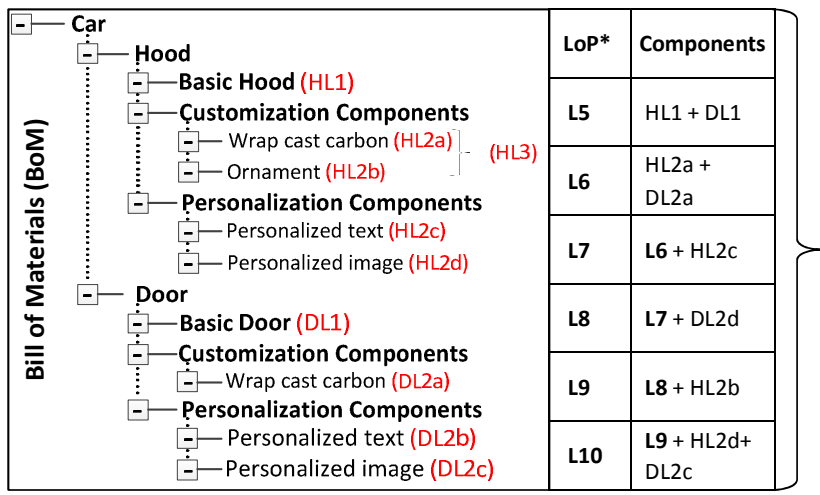

\begin{tabular}{|r|l|}
\hline \multicolumn{2}{|c|}{ Bill of Processes (BoP) } \\
\hline $\mathbf{1}$ & Hood manufacturing \\
\hline $\mathbf{2}$ & Door manufacturing \\
\hline $\mathbf{3}$ & Ornament manufacturing \\
\hline $\mathbf{4}$ & Wrap cast carbon manufacturing \\
\hline $\mathbf{5}$ & Personalised text printing \\
\hline $\mathbf{6}$ & Personalised image printing \\
\hline $\mathbf{7}$ & Apply wrap cast carbon on hood \\
\hline $\mathbf{8}$ & Apply wrap cast carbon on door \\
\hline $\mathbf{9}$ & Apply ornament \\
\hline $\mathbf{1 0}$ & Apply personalised text \\
\hline $\mathbf{1 1}$ & Apply personalised image \\
\hline
\end{tabular}

Fig. 2. BoM, BoP of the personalised car and LoPs

\section{Design and Planning Method}

The decision-making method includes resource-task assignments. The algorithms used in this procedure are either an Exhaustive Search (EXS) or the ISA. EXS generates the entire search space and identifies the best alternative. Thus, EXS is computationally intensive. ISA is an artificial intelligence search method that utilises three adjustable control parameters [15], namely: SNA (Selected Number of Alternatives) that controls the breadth of the search, DH (Decision Horizon) that controls the depth of the search and SR (Sampling Rate) that guides the search towards high quality paths.

The steps of the decision-making method are: a) form a set of alternatives, b) determine the decision-making criteria, c) calculate the utility value of alternatives, and d) select the best alternative [16][17]. Multiple conflicting criteria are considered simultaneously, based on the design and planning objectives. The best identified schemes are fed into the simulator. Fig. 3 depicts the input-output data and the workflow of the method. 


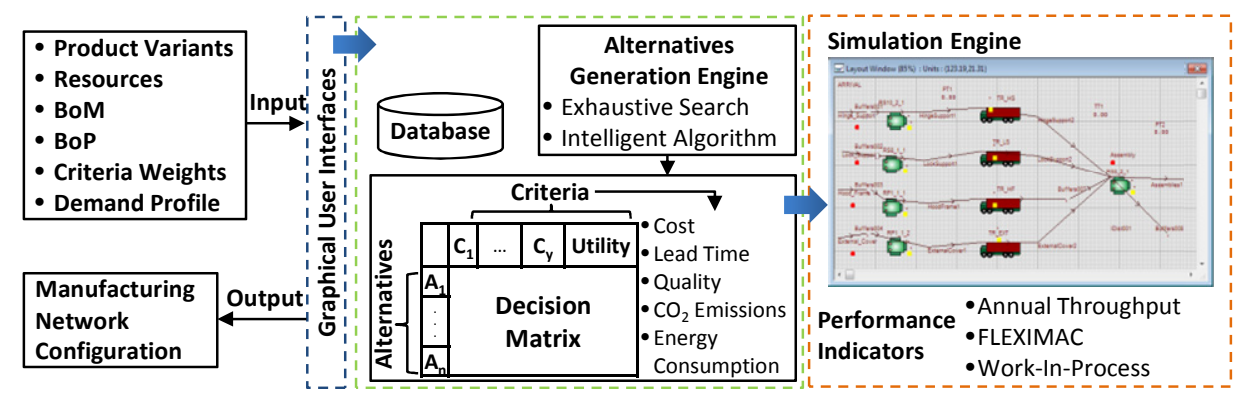

Fig. 3. Input-Output and Workflow of the presented approach

\subsection{Criteria}

The quality of the schemes is quantified by the means of the following criteria:

1. $\operatorname{Cost}(\mathbf{1})$ is the sum of the production and transportation cost (in $€$ ) [15][18]:

2. Lead time (2) is calculated from the point that an order is placed to the point that it is actually available for satisfying customer demand [15][19]:

3. Energy Consumption (3) takes into consideration the Watt specifications and the processing time of each manufacturing resource [18][20]:

4. $\mathrm{CO}_{2}$ Emissions (4) are calculated taking into consideration the distance travelled and the emitted grams of $\mathrm{CO}_{2}$ per kilometre [15][18][20][21]:

5. Quality (5) is an indicator that takes into account the mean quality of the parts, services that the manufacturing network partner provides and on their respect of due dates and takes values between, calculated based on empirical data [0-100].

6. Annual Production Rate (6) is expressed as the mean value of annual production volumes over the complete simulation period [7][19][22].

7. FLEXIMAC (7) is a quantification of flexibility, using the processing and flow time of the parts produced and is used to compare two similar networks. It is calculated using the system eigenvalues $\Omega_{\mathrm{i}}$ and computing the amplitude $\mathrm{Q}_{\mathrm{i}}$ on those $\Omega$ frequencies. It is then calculated as the average value of the ten largest $\mathrm{Q}_{\mathrm{i}}$ [7][15][22].

8. Work-In-Process (WIP) is the inventory between the start and end points of the manufacturing system without including raw materials and finished products [23].

Additionally, the following indicators are used to express the resource availability:

Mean Time Between Failures (MTBF) is defined for each one of manufacturing network partners. MTBF is based on previous manufacturing knowledge and historical data and takes into account the observed frequency that a supplier fails to deliver the ordered batch, because of resource break-downs or capacity constraints. Mean Time To Repair (MTTR): The MTTR is calculated for each one of the manufacturing network partners as the time required for them to resume production. 


$$
\begin{gathered}
C=\sum_{k=1}^{K} P c_{k}+\sum_{r=1}^{R} T r_{C r} \\
L=\sum_{k=1}^{K} P t_{k}+\sum_{r=1}^{R} T t_{r}+\sum_{k=1}^{K} S t_{k} \\
E C=E C_{T}+E C_{P}=\sum_{r=1}^{R} D_{r} * T C+\sum_{k=1}^{K} P t_{k} * R W_{k} \\
C E=\sum_{r=1}^{R} \frac{G * D_{r}}{N} \\
Q L=\frac{\sum_{k=1}^{K} Q L_{k}}{k} \\
A P=\frac{\sum_{i=1}^{n y} A P_{i}}{n_{y}} \\
F L E X I M A C=\frac{1}{10} \sum_{i=1}^{10} \frac{1}{2 Q_{i}}
\end{gathered}
$$

where: Pck: cost of task $\mathrm{k}(\mathrm{k}=1,2, \ldots \mathrm{K}), \mathrm{Tr}_{\mathrm{Cr}}$ : cost of route $\mathrm{r}(\mathrm{r}=1,2 \ldots \mathrm{R}), \mathrm{Pt}$ : processing time, $\mathrm{Tt}$ : transportation time, $\mathrm{St}$ : setup time, $\mathrm{EC}_{\mathrm{T}}$ : the sum of the energy due to transportation $(\mathrm{J})$, D: transportation distance $(\mathrm{km})$, TC: energy consumption per kilometre $(\mathrm{J} / \mathrm{km}) 21, \mathrm{EC}_{\mathrm{P}}$ : the sum of the energy for all the processes $(\mathrm{J}), \mathrm{RW}$ : the Watts of the resource $(\mathrm{J} / \mathrm{s})$, grams of $\mathrm{CO}_{2}$ emissions $/ \mathrm{Km} 21, \mathrm{~N}$ : number of products in one truck, $\mathrm{QL}_{\mathrm{k}}$ : the quality of the supplier that performs the task $\mathrm{k}$, $\mathrm{AP}_{\mathrm{i}}$ : the annual production volume for the $\mathrm{i}^{\text {th }}$ year of simulation and $\mathrm{n}_{\mathrm{y}}$ : the

\begin{tabular}{|c|c|c|c|c|c|c|c|c|c|c|c|c|}
\hline Month 1 & 2 & 3 & 4 & 5 & 6 & 7 & 8 & 9 & 10 & 11 & 12 & Total \\
\hline Orders 25.200 & 36.399 & 22.399 & 22.399 & 19.601 & 16.800 & 11.200 & 5.601 & 28.000 & 39.200 & 30.800 & 22.399 & $280 \times 10^{3}$ \\
\hline
\end{tabular}
number of years (simulation period), $\mathrm{Q}_{\mathrm{i}}$ : the eigenvalues of the system.

\section{$4 \quad$ Results and Discussion}

As a resource, a selection of machines that are responsible for performing a task (e.g. production of the basic hood) is assumed. The volatile demand profile, as provided by the automotive manufacturer for the simulation experiments, is depicted in Table 1.

Table 1. Demand profile of the car model of the case study

The computer simulation experiments were performed on an Intel $^{\mathrm{TM}}$ i7 $3.4 \mathrm{GHz}$ computer with $8 \mathrm{~GB}$ of RAM. Fig. 4 depicts that there is a strong correlation between the Total Number of Alternatives (TNA) and the required Computation Time (CT). The TNA especially in the case of L10 has a difference of 3 orders of magnitude from the L9 case. In the case of L8, the EXS execution was non-feasible due to computational constraints i.e. depletion of the available system memory. It is obvious that the ISA is the preferred method is cases with very large TNA. It is also noted that the CT for L9 and L10 is a projection based on the calculated TNA for these cases.

The values of the criteria the derived from the experiments are included in Fig. 5. Especially for the production of highly personalised products (L8), the Decentralised Manufacturing Network (DMN) depicts significantly reduced criteria values compared to the Centralised Manufacturing Network (CMN). In addition, the ISA yields results that belong to the $10 \%$ of the best solutions, in terms of utility value 

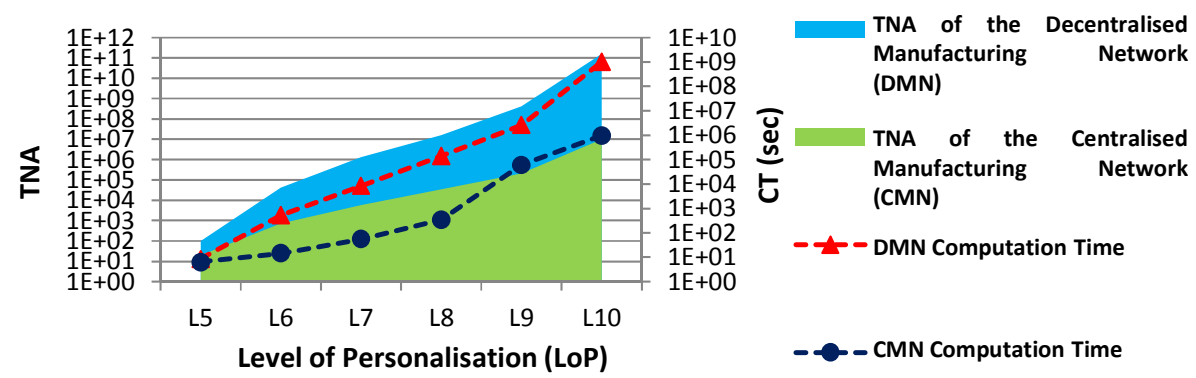

Fig. 4. TNA and CT for the CMN and DMN configurations for the different LoPs

(derived from the EXS). Indicatively, the ISA yielded a high quality solution with 23.3\% more cost, requiring however, one 1,026 times less CT than the EXS (pie-chart in Fig. 5). In realistic manufacturing cases, EXS is highly ineffective because TNA may be calculated in the order of billions. Thus, a timely and efficient solution can only be then obtained through the utilisation of the ISA. As a result, depending on the design and planning objectives, a trade-off between the time for obtaining the solution and its quality is necessary.

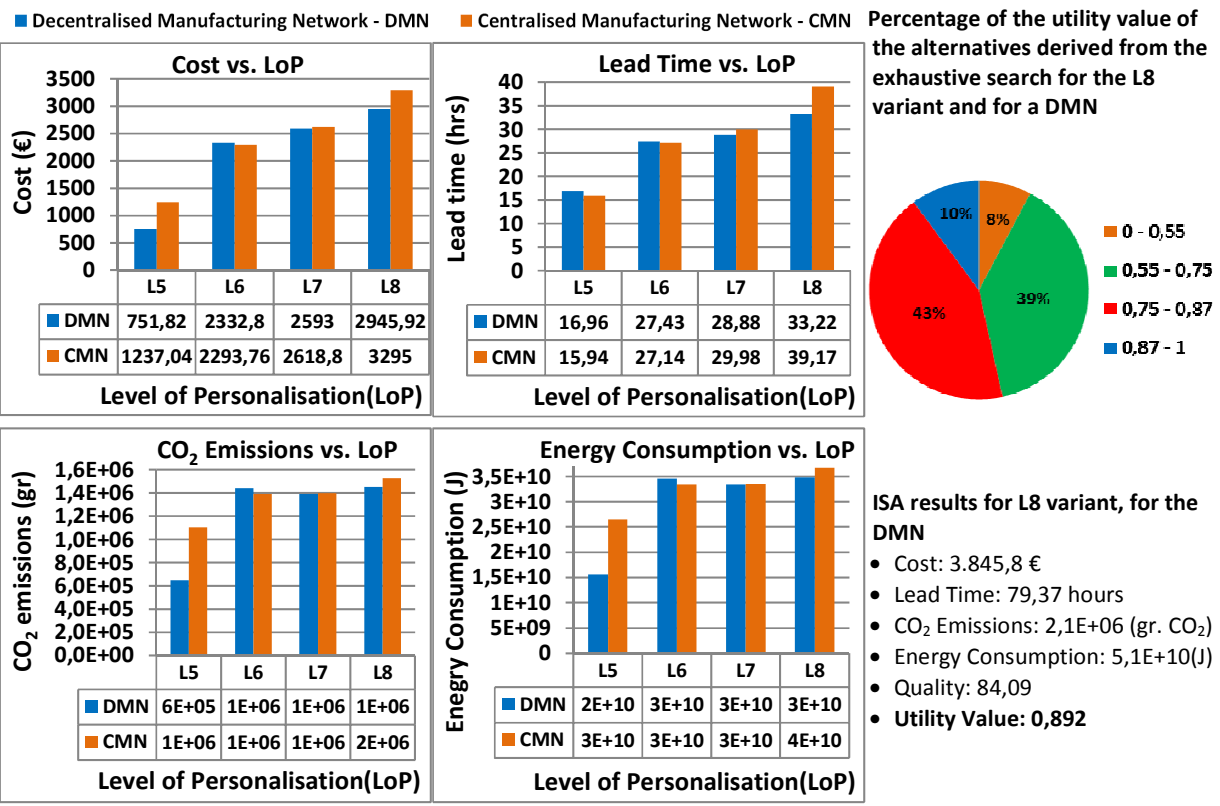

Fig. 5. Criteria Values and Utility Value of the EXS and ISA for the CMN and DMN and LoPs

The best configurations identified by the EXS were modelled in a simulator for the calculation of the flexibility, annual throughput and WIP (Fig. 6). The physical "as-is" manufacturing network was first modelled as a digital DES model. This initial model was verified through simulation and any bottlenecks were identified. Afterwards, the 
truck capacity as well as the buffer sizes were adjusted in all DES models, considering the volume of products and weight constraints. As a result, the transportation and storing activities were optimised in order to minimise idle times and improve machine utilisation. Finally, the initial stock was zero, thus, a ramp-up phase was included in all simulation experiments for the DMN and CMN and for the various LoPs.

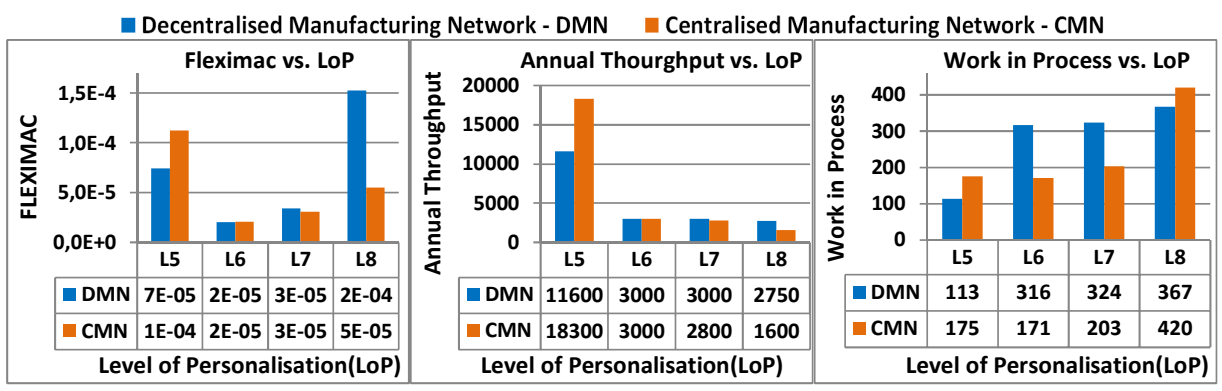

Fig. 6. Calculated Indicators for CMN and DMN configurations for the different LoPs

The simulation results depict that the flexibility indices of the CMN configurations are higher for non-customised products, whereas as the LoP increases, DMN displays higher flexibility. The annual throughput follows the same trend. For non-customised products, the CMN is more productive than the DMN ones and as the LoP increases the latter are more productive. The WIP is increasing as the LoP increases for both the $\mathrm{CMN}$ and $\mathrm{DMN}$, due to the fact that the number of required processes increases for the production of the final assembly.

\section{$5 \quad$ Conclusions and Future Work}

The work presented in this paper can support decision-makers during the design of efficient manufacturing network configurations focused on the production of personalised products. The computer simulation experiments depicted the advantages of the decentralised configurations, especially as the LoP increased. The calculated performance indicators of Annual Throughput, Flexibility and Work-in-Process revealed the superiority of the DMN configurations over the CMN under the same personalised product demand. For the case of non-customised products, the CMN configuration yielded better results, as was expected, because traditional CMN configuration were formed to satisfy the needs of mass production, where predictions regarding product demand were reasonably accurate. Moreover, the ISA supported the identification of high quality solutions in cases were an EXS was not feasible to be performed due to the required computation burden. More specifically, the EXS, in the case of the fully personalised product (L8 variant), yielded a result in 2.340 minutes, a non-optimum scenario for real-life manufacturing terms. On the other hand, the ISA yielded high quality results with a deviation of $10,39 \%$ in the utility 
value from the best solution obtained from EXS in 3 minutes, a difference of 3 orders of magnitude. Future work will focus on extending the capabilities of the method. At first the procedure of feeding alternative manufacturing network configurations (DES models) in the simulation engine will be automated. Moreover, additional flexibility indicators will be used for assessing the alternative network configurations. A webbased mechanism for the automatic calculation of the distances between the autonomous manufacturing network entities will be incorporated. Finally, the method will be deployed into a web-based tool.

Acknowledgments. This work has been partially supported by the EC funded project "e-CUSTOM - A Web-based Collaboration System for Mass Customisation" (NMP2SL-2010-260067).

\section{References}

1. Ueda, K., Takenaka, T., Váncza, J., Monostori, L.: Value creation and decision-making in sustainable society. CIRP Annals-Mfg. Tech. 58, 681-700 (2009)

2. The Economist: The Third Industrial Revolution, April 21-27 (2012)

3. Thirumalai, S., Sinha, K.: Customisation of the online purchase process in electronic retailing and customer satisfaction: An online field study. J. of Oper. Mgmt. 29, 477-487 (2011)

4. Radke, A.M., Tseng, M.M.: A risk management-based approach for inventory planning of engineering-to-order production. CIRP Annals-Mfg. Tech. 61, 387-390 (2012)

5. Herrmann, I.T., Hauschild, M.Z.: Effects of globalisation on carbon footprints of products. CIRP Annals-Mfg. Tech. 58, 13-16 (2009)

6. Tolio, T., Urgo, M.: A Rolling Horizon Approach to Plan Outsourcing in Manufacturingto-Order Environments Affected by Uncertainty. CIRP Annals-Mfg. Tech. 56, 487-490 (2007)

7. Michalos, G., Makris, S., Mourtzis, D.: An intelligent search algorithm-based method to derive assembly line design alternatives. Int. J. of Comp. Int. Mfg. 25, 211-229 (2012)

8. Labarthe, O., Espinasse, B., Ferrarini, A., Montreuil, B.: Toward a methodological framework for agent-based modelling and simulation of supply chains in a mass customisation context. Sim. Mod. Pr. and Th. 15, 113-136 (2007)

9. Persson, F., Araldi, M.: The development of a dynamic supply chain analysis tool: Integration of SCOR and discrete event simulation. Int. J. of Prod. Econ. 121, 574-583 (2009)

10. Pierreval, H., Bruniaux, R., Caux, C.: A continuous simulation approach for supply chains in the automotive industry. Sim. Mod. Pr. and Th. 15, 185-198 (2007)

11. Longo, F., Mirabelli, G.: An advanced supply chain management tool based on modelling and simulation. Comp. \& Ind. Eng. 54, 570-588 (2008)

12. Chiang, W.C., Russell, R., Xu, X., Zepeda, D.: A simulation/metaheuristic approach to newspaper production and distribution supply chain problems. Int. J. of Prod. Econ. 121, 752-767 (2009)

13. Garey, M., Johnson, D.: Computers and Intractability - A Guide to the Theory of NPCompleteness, 1st edn. W.H.Freeman \& Co Ltd., New York (1990)

14. Yoo, T., Cho, H., Yucesan, E.: Hybrid algorithm for discrete event simulation based supply chain optimisation. Exp. Sys. With Appl. 37, 2354-2361 (2010) 
15. Mourtzis, D., Doukas, M., Psarommatis, F.: A multi-criteria evaluation of centralised and decentralised production networks in a highly customer-driven environment. CIRP Annals-Mfg. Tech. 61, 427-430 (2012)

16. Chryssolouris, G.: Manufacturing Systems - Theory and Practice, 2nd edn. Springer (2006)

17. Chryssolouris, G., Dicke, K., Lee, M.: On the Resources Allocation Problem. Int. J. of Prod. Res. 30, 2773-2795 (1992)

18. Mourtzis, D., Doukas, M., Psarommatis, F.: Design and planning of decentralised production networks under high product variety demand. Procedia CIRP (2012), doi: http://dx.doi.org/10.1016/j.procir.2012.07.051

19. Liao, C.J., Shyu, C.H.: An Analytical Determination of Lead Time with Normal Demand. Int. J. of Oper. \& Prod. Mgmt. 11, 72-78 (1991)

20. Mourtzis, D., Doukas, M., Psarommatis, F.: Environmental impact of centralised and decentralised production networks in the era of personalisation. Lect. Notes in Prod. Eng, vol. 1, pp. 396-408 (2012), doi:10.1007/978-3-642-30749-2

21. EPA (2010), http: / /www. epa.gov

22. Alexopoulos, K., Papakostas, N., Mourtzis, D., Gogos, P., Chryssolouris, G.: Quantifying the flexibility of a manufacturing system by applying the transfer function. Int. J. of Comp. Int. Mfg. 20, 538-547 (2006)

23. Hopp, W.J., Spearman, M.L.: Factory Physics: Foundations of Manufacturing Management, 2nd edn. Richard D. Irwin (1995) 